\title{
PAPR Reduction Using Precoding Technique along with ICTF in OFDM Systems
}

\author{
Naveed Showkat and Sandeep Kumar Arora* \\ ${ }^{1}$ Discipline of Electronics and Communication Engineering \\ Lovely Professional University, Jalandhar, Punjab, India-144411 \\ ${ }^{2}$ Discipline of Electronics and Communication Engineering \\ Lovely Professional University, Jalandhar, Punjab, India-144411 \\ E-mail: ${ }^{1}$ naveedjan1914@gmail.com, ${ }^{2}$ sandeep.16930@lpu.co.in
}

\begin{abstract}
An Iterative Companding Transform and Filtering (ICTF) is an efficient technique which is integrated with precoding technique for minimization of PAPR (Peak-to-Average Power Ratio) for OFDM signal (Orthogonal Frequency Division Multiplexing). With the help of specifically designed duplication procedure, ICTF with Precoding Technique is able to perform significant minimization of PAPR. In the presented paper, an inclusive hypothetical analysis is shown along with nearly valuable results such as PAPR gain is derived. Compared to the simple companding with precoding technique, ICTF with Precoding Technique can dramatically decrease the desired PAPR with low complexity.
\end{abstract}

Keywords: OFDM, PAPR, Companding transform and filtering, Zadoff Chu matrix

\section{Introduction}

Under severe channel conditions and its inherent error resistance OFDM is widely implemented over wideband wireless. Orthogonal frequency division multiplexing (OFDM) is a widely used modulation and multiplexing technology, which has become the basis of many telecommunications standards including wireless local area networks (LANs), digital terrestrial television (DTT) and digital radio broadcasting in much of the world. In the past, as well as in the present, the OFDM is referred in the literature as Multi-carrier, Multi-tone and Fourier Transform. The OFDM concept is based on spreading the data to be transmitted over a large number of carriers, each being modulated at a low rate. The carriers are made orthogonal to each other by appropriately choosing the frequency spacing between them. A multicarrier system, such as FDM (Frequency Division Multiplexing), divides the total available bandwidth in the spectrum into subbands for multiple carriers to transmit in parallel. [15] It combines a large number of low data rate carriers to construct a composite high data rate communication system. Orthogonality gives the carriers a valid reason to be closely spaced with overlapping without ICI. [16]

In contrast to conventional Frequency Division Multiplexing, the spectral overlapping among subcarriers are allowed in OFDM since orthogonality will ensure the subcarrier separation at the receiver, providing better spectral efficiency and hence the use of steep band pass filter is eliminated. OFDM transmission system offers possibilities for alleviating many of the problems encountered with single carrier systems. It has the advantage of spreading out a frequency selective fade over many symbols. This effectively randomizes burst errors caused by fading or impulse interference so that instead of several adjacent symbols being Completely destroyed, many symbols are only slightly distorted. This allows successful reconstruction of majority of them even without forward error correction. Because of dividing an entire signal bandwidth into many narrow sub bands, the frequency response over individual sub bands is relatively flat due 
to sub band are smaller than coherence bandwidth of the channel. Thus, equalization is potentially simpler than in a single carrier system and even equalization may be avoided altogether if Differential encoding is implemented.

\subsection{Principle of OFDM}

In digital communications, information is expressed in the form of bits. The term symbol refers to a collection, in various sizes, of bits. OFDM data are generated by taking symbols in the spectral space using M-PSK, QAM, etc, and convert the spectra to time domain by taking the Inverse Discrete Fourier Transform (IDFT). Since Inverse Fast Fourier Transform (IFFT) is more cost effective to implement, it is usually used instead [16]. The main features of a practical OFDM system are as follows:

- - Some processing is done on the source data, such as coding for correcting errors, interleaving and

- mapping of bits onto symbols. An example of mapping used is QAM.

- - The symbols are modulated onto orthogonal sub-carriers. This is done by using IFFT.

- Orthogonality is maintained during channel transmission. This can be achieved by adding a cyclic

- prefix to the OFDM frame to be sent. The cyclic prefix consists of the L last samples of the frame, which are copied and placed in the beginning of the frame. It must be longer than the channel impulse response.

- Synchronization: cyclic prefix can be used to detect the start of each frame. This is done by using

- the fact that the L first and last samples are the same and therefore correlated.

- Demodulation of the received signal by using FFT.

- Channel equalization: the channel can be estimated either by using a training sequence or sending

- known so-called pilot symbols at predefined sub-carriers.

- Decoding and de-interleaving.

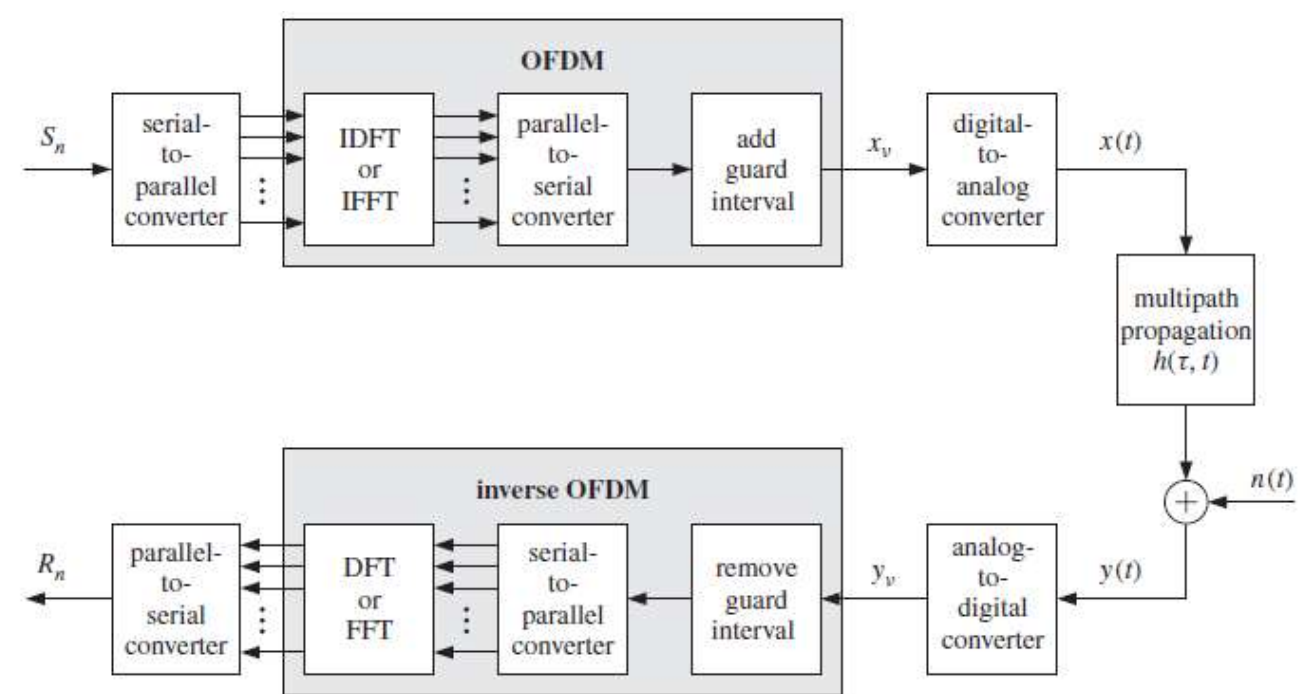

Figure 1. Digital Multicarrier Transmission System Applying OFDM 
IDFT: Inverse Discrete Fourier Transform

IFFT: Inverse Fast Fourier Transform

Sn: Input Signal

Rn: Output Signal

\subsection{Peak to Average Power Ratio (PAPR)}

But due to certain critical issues system suffers with shortcomings like, greater PAPR (Peak to Average Power Ratio). This problem produces an inter-modulation between out of band interference (OBI) and sub carriers [1]. In order to overcome these shortcomings Digital to Analog (D/A) converter and high power amplifier are used with its dynamic range for avoidance of nonlinear distortion [2]. In turn numerous techniques are suggested for the reduction of PAPR, such as [3], Clipping and filtering [4], Selective Mapping [5], partial transmit sequence (PTS) [6], tone reservation [7] and companding transform [8]. Amongst these techniques, clipping and filtering is preferable but it reduces BER (Bit error rate) due to OBI problem and hence degrades the performance of OFDM system. It is observed that $\mathrm{OBI}$ in comparison with in-band distortion is further critical and hinders the radio communication [4].

The traditional CT technique is the solution of OBI problem [8], which makes usage of soft compression for clipping peaks of signal and generating less OBI problem. Immunization from the channel noise can be easily obtained by intentional expansion of large signals and compression of smaller ones. Due to CT's robust and simplified nature, it is used extensively. It can be used in with no restriction on certain parameters like sub carrier number, frame format and constellation types. Prior focus of CT method was designing of linear and nonlinear companding profile. Succeeding to it the statistical distribution in OFDM was explored. [9]. Under linear and nonlinear CT types certain methods are advocated which are $\mu$-law companding [8] and Exponential Companding technique. Although CT is similar to clipping, but it possesses pre-distortion processing which is applied over original signal. In the existing CT methods, a trade-off is observed between BER and PAPR.

Two issues are observed from the previous CT techniques. Foremost is how to attain an actual trade-off between PAPR reduction and BER enhancement. Secondly is how to reduce the OBI problem. In the proposed paper, an ICTF (Iterative CT and filtering) method is suggested which is an iterative filtering approach in ICF method whose main objective is to reduce PAPR of OFDM signal.

With usage of an iterative procedure, ICTF can allow user to obtain a significant reduction in PAPR as well as simultaneous improvement in BER performance. Moreover, to reduce OBI problem a frequency domain filtering is designed for minimization of OBI spectral re-growth. With comparison to traditional $\pi$ method, ICTF provides desired PAPR with less computational intricacy.

Precisely, it is presented that ICTF without de-companding operation provides a good BER performance at the receiver side. The effect of ICTF is shown through theoretical analysis and adequate numerical simulations.

\section{Proposed Companding Transform with Precoding Technique}

The common formulae and fundamental process of companding and precoding technique are hereby shown in this section.

\subsubsection{Iterative Companding transform}

The main aim of ICTF technique is to attain a remarkable PAPR reduction. In ICTF technique shown in Figure 1, a repetitive companding will be done as long as the obtained PAPR value is not less than that of the threshold value. 


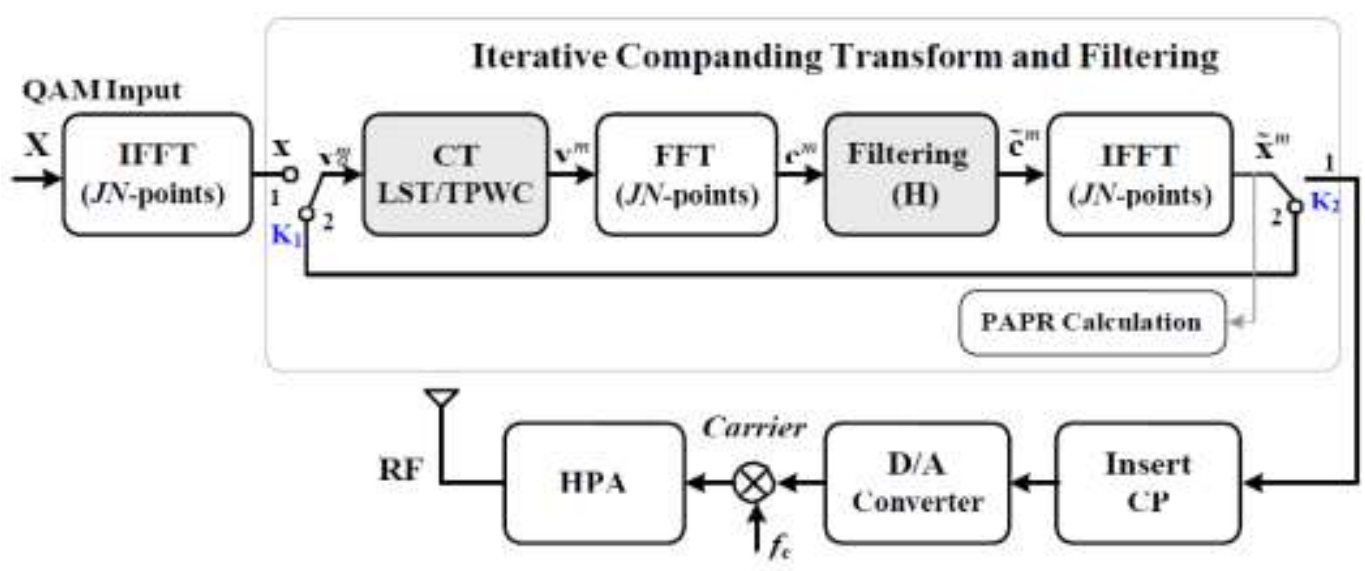

Figure 2. Iterative Companding Transform and Filtering

IFFT: Inverse Fast Fourier Transform

CT: Companding Transform

D/A: Digital to Analog converter

HPA: High Power Amplifier

As seen from the Figure 1 that there is a companding block. Here are some of the companding techniques that can be used:

\subsubsection{Linear Symmetrical Transform}

Linear Symmetrical Transform (LST) is one of the CT method, where the companding function is given as follows:

$$
F(x)=(k \cdot|x|+b) \cdot \operatorname{sgn}(x)
$$

here sgn (.) is a sign function, and two parameters $0<\mathrm{k}<\square 1$ and $\mathrm{b}>0$ are used for specification of companding profile.

\subsubsection{Two-Piecewise Companding}

It has been proved that out of linear and nonlinear CT methods, Linear NonSymmetrical Transform (LNST) is suitable in reduction of PAPR and improvement of BER performance. Furthermore, Two-Piecewise Companding (TPWC) [12] is an improved LNST to reduce the 'abrupt jump' problem, and its companding function is shown as follows:

$$
\begin{array}{cc}
\mathrm{U} 1|\mathrm{x}|) * \operatorname{sgn}(\mathrm{x}) & |\mathrm{x}| \leq \mathrm{V} \\
\left(\mathrm{U}_{2}|\mathrm{x}|+\mathrm{s}\right) * \underset{\operatorname{sgn}(\mathrm{x})}{ } & |\mathrm{x}|>\mathrm{V} \\
f(x)= &
\end{array}
$$

where $\mathrm{u} 1>1,0<\mathrm{u} 2<1, \mathrm{~s}=(\mathrm{u} 1-\mathrm{u} 2) \mathrm{v}>0$ and $0 \leq \mathrm{v} \leq \mathrm{V}$ is the cutoff point with $\mathrm{V}=\max (|\mathrm{xn}|)$ for $0 \leq \mathrm{n} \leq \mathrm{JN}-1$

\subsection{4. $\mu$-law Compander}

The $\mu$-law compander allocates the logarithmic function at the transmitter portion. Generally, $\mu$-law compression characteristic is expressed as:

$$
y=\log e\left(1+\mu \frac{|x|}{v}\right) \cdot \operatorname{sgn}(x) \div \log e(1+\mu)
$$




\subsubsection{A-Law Companding}

A-Law is the recommended companding standard used across the Europe.

The equation is written as:

$$
F(x)=\operatorname{sgn}(x) \cdot A|x| \div(1+\ln A) \quad 0 \leq\|x\| \leq 1 \div A
$$

Where A: companding parameter

$\mathrm{X}$ : normalized integer to be companded.

\subsection{Precoding Technique}

In the following section precoding techniques are given which is used to get a signal with minimized PAPR unlike the situation of conventional OFDM without precoding techniques and to decrease the interference which is formed by multiple users in the OFDM system. It is of prior importance that the PAPR reduction techniques must compensate the nonlinearities of the HPA (High Power Amplifier). Some precoding techniques are shown as follows:

\subsubsection{Zadoff-Chu Matrix Transforms (ZCT)}

Zadoff-Chu codes are the one of the distinct case of the generalized Chip-Like polyphase sequences that has optimal correlation properties [13] [14]. The Zadoff-Chu are complex- valued sequences, when these sequences are applied to the radio signal and gives an electromagnetic signal with constant amplitude. Zadoff-Chu matrix transform can be applied for OFDM as shown in Figure 2. These sequences are class of poly phase sequences which possess optimal correlation properties and have an ideal periodic autocorrelation and constant magnitude. When cyclically the shifted version of the sequence is imposed on a signals result in zero cross-correlation. Zadoff- Chu matrix transform pre-coding is introduced in this paper which would diminish the PAPR value of any OFDM systems. This technique uses Zadoff-Chu sequences of length $\mathrm{L}$ which can be define as equation (6 and 7). the nonlinearities of the HPA. Some pre-coding techniques are presented in the following.

$$
\begin{array}{cr}
e^{j 2 \pi r \div L \times(k \div 2+q k)} & \text { for L even } \\
e^{j 2 \pi r \div L \times(k \div 2+q k)} & \text { for L odd } \\
e^{j 2 \pi r \div L \times(k(k+1) \div 2+q k)} & \text { for L odd } \\
\mathrm{Z}(\mathrm{k})= & \mathrm{Z}(\mathrm{k})=
\end{array}
$$

\subsubsection{Walsh-Hadamard Transform (WHT)}

The Walsh-Hadamard Transform (WHT) is an orthogonal and non-sinusoidal technique which would decompose a signal into sets of basis function. These basis function are called as Walsh function. When linked with the original signal the Hadamard transform scheme can reduce the presence of high peaks. Usage of Hadamard transform would reduce autocorrelation of input sequence leading to a decrease in the value of PAPR. Moreover, it does not require any side information to be transmitted to the reception side. Walsh-Hadamard Transform can be applied for OFDM as shown in Figure 2. In the subsequent section a brief review of Hadamard transform is given where $\mathrm{H}$ represents Hadamard transform matrix of $\mathrm{N}$ orders and Hadamard matrix is standardized orthogonal matrix. The Hadamard matrix of 2 orders is given by:

$$
\begin{array}{ccc}
\mathrm{H}_{2}=1 / \sqrt{ } 2 & 1 & 1 \\
1 & -1 &
\end{array}
$$




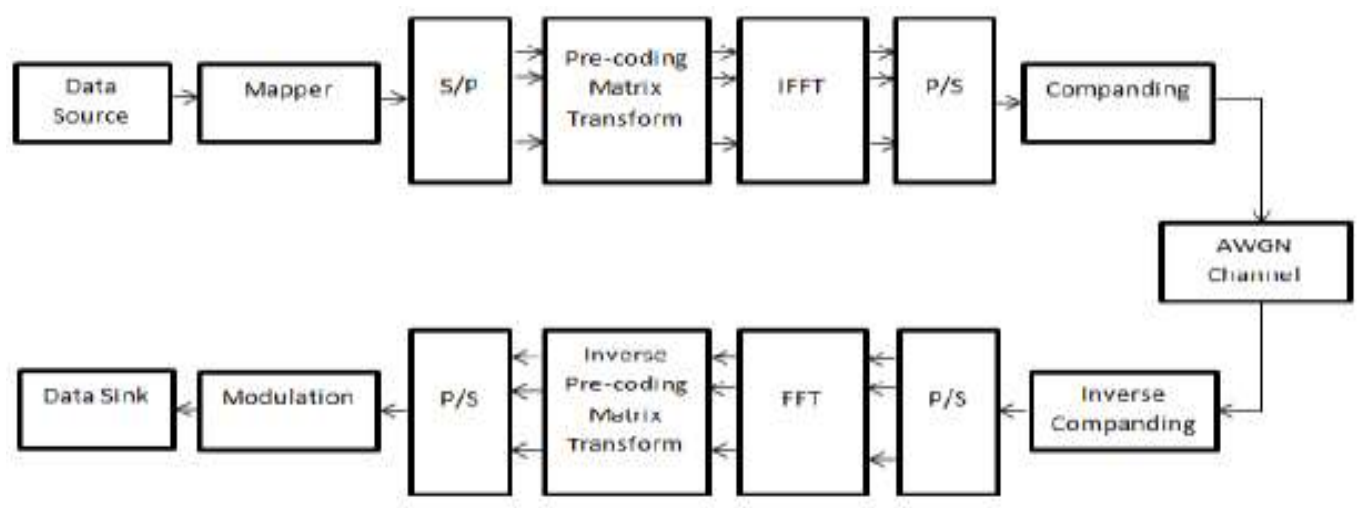

Figure 3. Precoding Matrix Used for PAPR Reduction

S/P: Serial to Parallel Converter

IFFT: Inverse Fast Fourier Transform

$\mathrm{P} / \mathrm{S}$ : Parallel to Serial converter

\section{Proposed Technique}

The new technique proposed is the Iterative Companding Transform with Filtering combined with the Precoding matrix as shown in the Figure 3. In this technique, firstly a symbol is passed through precoding matrix and then, the signal is processed through iterative companding and filtering transform. The desired PAPR is already set and if the PAPR calculated is found to be greater than that of the desired PAPR then the symbol is sent back to the Iterative loop.

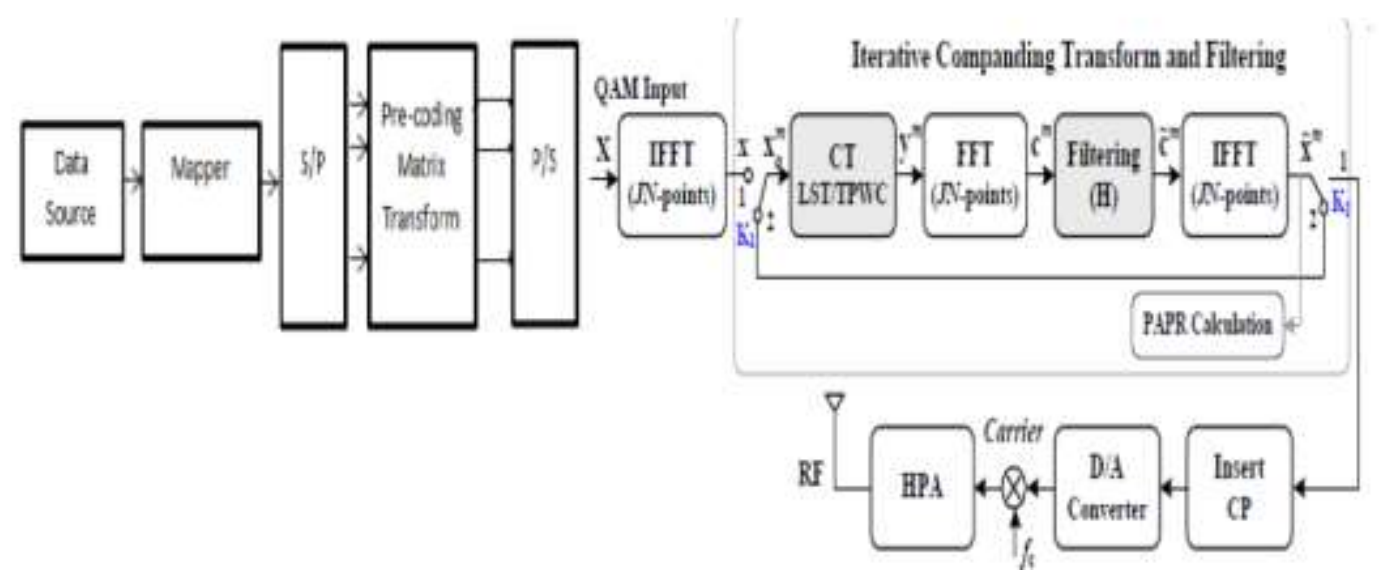

Figure 4. Precoding Matrix Combined with ICTF

\section{Simulation and Results}

As seen in Figure 5 the PAPR using ZCT technique is decreased to the value of almost $5 \mathrm{db}$ but if we compare the same result in Figure 6 in which PAPR is calculated using Precoding with ICTF technique, it is seen that the PAPR decreases significantly. 


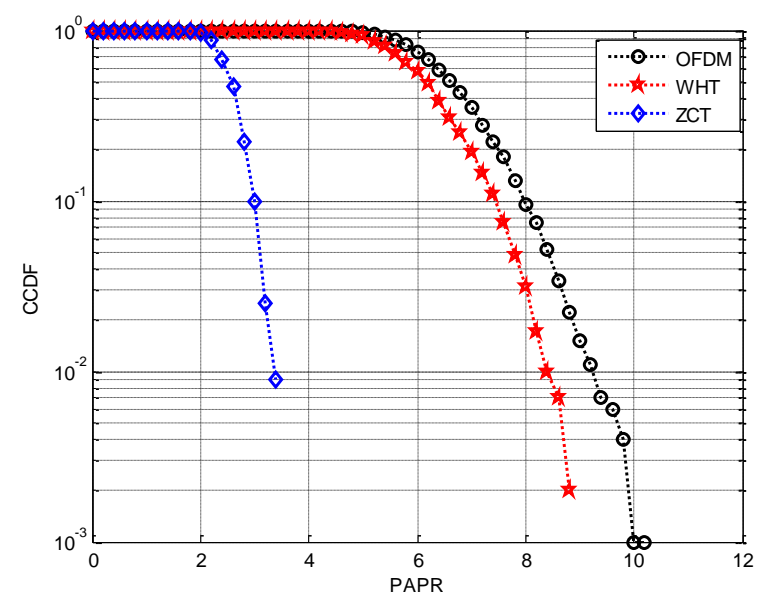

Figure 5. PAPR with A-Law Companding

Now, as we can see in the Figure 6 below that the PAPR almost is decreased by the $8 \mathrm{db}$. Thus, it is noted that the technique of using Precoding with ICTF is more effective in reducing PAPR in comparison with the existing technique of simple companding with Precoding matrix.

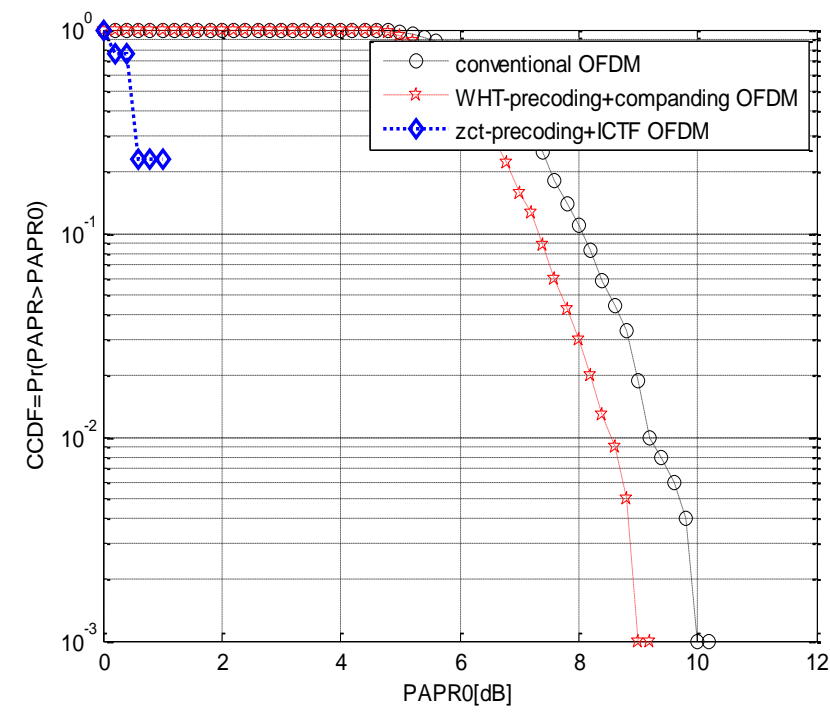

Figure 6. PAPR with ICTF Companding

\section{Conclusion and Future Scope}

We analyzed that the ICTF technique used with Precoding technique is more effective than using the simple companding technique along with Precoding technique. Thus, there is no need for HPA, as PAPR is reduced significantly. A lot of attention is required in the channel estimation as it degrades the performance of entire OFDM system. Better tradeoff between BER and PAPR can be further obtained. In future, MIMO OFDM concepts can be used for decreasing the PAPR. The demand for high data rate wireless communication has been increasing drastically over the last decade. One way to transmit this high data rate information is to employ well-known conventional single carrier systems. Since the transmission bandwidth is much larger than the coherence bandwidth of the channel, 
highly complex equalizers are needed at the receiver for accurately recovering the transmitted information. Multi-carrier techniques can solve this problem significantly. In this paper we have discussed about the basic idea behind the OFDM, the most emerging technology of this era. Here we take a review on its concept, its properties in terms of its advantages and disadvantages, its limitations and also its applications in different fields.

\section{References}

[1] Y Yang, C. Yang, B. Ai, "Iterative Companding Transform and Filtering for Reducing PAPR of OFDM Signal," IEEE Transactions on Cosumer Electronics., vol.61, no. 2, (2015), pp. 144-150.

[2] B. Ai, Z. Yang, C. Pan, T. Zhang, and J. Ge, "Effects of PAPR reduction on HPA predistortion," IEEE Trans. Consumer Electron., vol. 51, no. 4, (2005), pp. 1143-1147.

[3] Y. Rahmatallah, and S. Mohan. "Peak-to-average power ratio reduction in OFDM systems: A survey and taxonomy," IEEE Commun Surveys \& Tutorials, vol. 15, no.4, (2013), pp. 1567-1592.

[4] L. Wang and C. Tellambura, "A simplified clipping and filtering Technique for PAR reduction in OFDM Systems,” IEEE Signal Process. Lett., vol. 12, no. 6, (2005), pp. 453-456.

[5] J. Yang, L. Chen, Q. Liu, and D. Chen, "A modified selected mapping Technique to reduce the peak-toaverage power ratio of OFDM signals," IEEE Trans. Consumer Electron. vol. 53, no. 3, (2007), pp. 846-851.

[6] P. Varahram and B. M. Ali, "Partial Transmit Sequence Scheme with New Phase Sequence for PAPR Reduction in OFDM Systems," IEEE Trans. On Consumer Electron., vol. 57, no. 2, (2011), pp. 366371.

[7] Youngin Park, Sangchae Lim, Dongsoo Har, "Adaptive Phase Rotation of OFDM Signals for PAPR Reduction,” IEEE Trans. on Consumer Electron., vol. 57, no. 4, (2011), pp. 1491-1495.

[8] X. B.Wang, T. T. Tjhung, and C. S. Ng, "Reduction of peak-to-average power ratio of OFDM system using a companding technique," IEEE . Song, "Exponential companding transform for PAPR reduction in OFDM systems," IEEE Trans. Broadcast., vol. 51, no. 2, (2005), pp. 244-248.

[9] Yuan Jiang, "New Companding Transform for PAPR Reduction in OFDM," IEEE Communications, vol. 14, no. 4, (2010), pp. 282-284.

[10] T. Jiang, Y. Yang, and Y. Song, "Exponential companding transform for PAPR reduction in OFDM systems," IEEE Transactions for Broadcast., vol. 51, no. 2, (2005), pp. 244-248.

[11] Yuan Jiang, "New Companding Transform for PAPR Reduction in OFDM," IEEE Communicarions Lett., vol. 14, no. 4, (2010), pp. 282-284.

[12] P. Yang, A. Hu, "Two-piecewise companding transform for PAPR reduction of OFDM signals," in Proc. IEEE International Conference on Wireless Communications and Mobile Computing, Istanbul, Turkey, (2011), pp. 619-623.

[13] I.Baig and V. Jeoti " PAPR Reduction in OFDM Systems: Zadoff-Chu Matrix Transform Based Pre/Post-Coding Techniques, (2010), pp.475-481.

[14] S. B. Slimane, "Reducing the peak-to-average power ratio of OFDM signals through precoding," IEEE Trans. Vehicular Technology, vol. 56, no. 2, (2007), pp. 686-695.

[15] Theory of Frequency Division Multiplexing: http://zone.ni.com/devzone/cda/ph/p/id/269

[16] Acosta, Guillermo. “OFDM Simulation Using MATLAB” 2000

\section{Authors}

Naveed Showkat, is M. TECH in Department of ECE at Lovely Professional University, Jalandhar. His research interests include Optical Networks, OFDM and Wireless Sensor Networks.

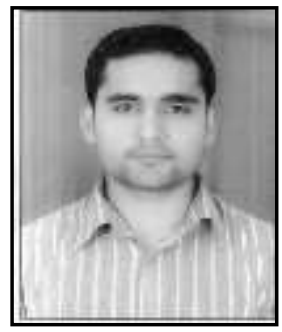

Sandeep Kumar Arora, is currently pursuing $\mathrm{Ph}$. D. in Electronics \& Electrical Engineering with Spl. in Design of Secure Initiation Protocol in VANET. He is working as an Asst. Prof. in Lovely Professional University since 2011. His research interest includes Wireless Sensor Networks, Computer Networks, Adhoc Networks Communications and Cryptography. He is a member of IEEE and also the author of more than one dozen research papers indexed in Scopus. 